UFIFT-QG-15-05

\title{
Fine Tuning May Not Be Enough
}

\author{
S. P. Miao ${ }^{1 *}$ and R. P. Woodard ${ }^{2 \dagger}$ \\ 1 Department of Physics, National Cheng Kung University \\ No.1, University Road, Tainan City 70101, TAIWAN \\ 2 Department of Physics, University of Florida \\ Gainesville, FL 32611, UNITED STATES
}

\begin{abstract}
We argue that the fine tuning problems of scalar-driven inflation may be worse than is commonly believed. The reason is that reheating requires the inflaton to be coupled to other matter fields whose vacuum fluctuations alter the inflaton potential. The usual response has been that even more fine-tuning of the classical potential $V(\varphi)$ can repair any damage done in this way. We point out that the effective potential in de Sitter background actually depends in a complicated way upon the dimensionless combination of $\varphi / H$. We also show that the factors of $H$ which occur in de Sitter do not even correspond to local functionals of the metric for general geometries, nor are they Planck-suppressed.
\end{abstract}

PACS numbers: 04.62.+v, 98.80.Cq, 12.20.Ds

* e-mail: spmiao5@mail.ncku.edu.tw

† e-mail: woodard@phys.ufl.edu 


\section{Introduction}

It is desirable to define inflation as a phase of accelerated expansion, distinct from any particular mechanism for achieving it. The explanatory power of an epoch accelerated expansion during the very early universe was realized in the late 1970's and early 1980's [1, 2, 3, 4, 5. The case for primordial inflation is now so strong as to be nearly incontrovertible [6, 7]. However, there is no similarly compelling indication for what caused it to occur.

The earliest, and still the simplest, model of inflation is general relativity with a minimally coupled scalar inflaton $\varphi(x)$ [8, 9, 10],

$$
\mathcal{L}=\frac{R \sqrt{-g}}{16 \pi G}-\frac{1}{2} \partial_{\mu} \varphi \partial_{\nu} \varphi g^{\mu \nu} \sqrt{-g}-V(\varphi) \sqrt{-g} .
$$

Models of this type certainly work because there is constructive procedure for determining the potential $V(\varphi)$ needed to support any expansion history [11, 12, 13, 14, 15]. However, there are six fine-tuning problems:

1. Initial Conditions - Inflation will not begin unless the inflaton is approximately homogeneous, with more potential energy than kinetic energy, over more than a Hubble volume [16, 17, 18];

2. Duration - Inflaton will not last long enough (more than 50 e-foldings) unless $V(\varphi)$ is very flat [8, 9];

3. Scalar Perturbations - The magnitude of scalar perturbations will not agree with observations unless $(G V)^{3} / V^{\prime 2} \sim 10^{-11}$ [19];

4. Tensor Perturbations - The magnitude of tensor perturbations will be too large unless $V^{\prime 2} / G V^{2} \lesssim 1$ [19];

5. Reheating - The post-inflationary universe will not reach a hot enough temperature unless the inflaton couples to ordinary matter because its gravitational couplings do not suffice [20];

6. Cosmological Constant - The post-inflationary universe will not evolve correctly unless the minimum of the scalar potential obeys $G^{2} V_{\min } \approx$ $10^{-123}$ [21, 22, 23, 24].

Some of these conditions work against one another. In particular, the matter couplings required by (5) induce Coleman-Weinberg corrections of 
the form $\pm \varphi^{4} \ln (\varphi)$ which disturb conditions (2-4) and necessitate additional fine tuning. The staggering amount of fine-tuning which is required disturbs many cosmologists [25, 26, 27, 28].

However distasteful all this fine tuning might seem, it has always been believed that the thing could at least be done. The purpose of this paper is to point out that this may not be true: the matter-inflaton couplings which are required by (5) actually induce gravitational couplings which are not Planck-suppressed and are not even local. Because allowed modifications of the action are restricted to be local, these nonlocal corrections to the inflaton effective potential cannot be eliminated. In section 2 we describe the true form of the effective potentials which emerge on de Sitter background from other scalars, from Yukawa fermions and from gauge particles. In section 3 we argue that the complicated factors of the de Sitter Hubble constant $H$ which appear in these results are not local functionals of the metric for a general cosmological geometry. Section 4 discusses the practical problem.

\section{Effective Potentials for de Sitter}

The purpose of this section is to give the one loop quantum corrections to the inflaton potential arising from hypothetical couplings to other scalars, to a massless fermion, and (for a charged inflaton) to a vector gauge boson. In each case we first give a formal expression for the result in terms of the unknown coincidence limit of the propagator for massive scalars with arbitrary conformal coupling,

$$
\left[\square-\xi R-M^{2}\right] i \Delta\left[\xi, M^{2}\right]\left(x ; x^{\prime}\right)=\frac{i \delta^{D}\left(x-x^{\prime}\right)}{\sqrt{-g}},
$$

where $R$ is the Ricci scalar and $\square$ is the covariant scalar d'Alembertian $\sqrt{-g} \mathbf{\square} \equiv \partial_{\mu}\left(\sqrt{-g} g^{\mu \nu} \partial_{\nu}\right)$. The only inflationary geometry for which the coincidence limit of this propagator is known for $M \neq 0$ is de Sitter,

$$
\left.i \Delta\left[\xi, M^{2}\right](x ; x)\right|_{\text {de Sitter }}=\frac{H^{D-2}}{(4 \pi)^{\frac{D}{2}}} \frac{\Gamma\left(\frac{D-1}{2}+\nu\right) \Gamma\left(\frac{D-1}{2}-\nu\right) \Gamma\left(1-\frac{D}{2}\right)}{\Gamma\left(\frac{1}{2}+\nu\right) \Gamma\left(\frac{1}{2}-\nu\right)},
$$

where $\nu^{2} \equiv\left(\frac{D-1}{2}\right)^{2}-D(D-1) \xi-\frac{M^{2}}{H^{2}}$ and $D$ is the dimension of spacetime, which is kept arbitrary to facilitate dimensional regularization. In each case (scalar, fermion and vector) we give the full de Sitter result, along with the large field and small field expansions. 


\subsection{Coupling to another scalar}

The inflaton might couple to another scalar $\phi(x)$, which need not be minimally coupled to gravity,

$$
\mathcal{L}_{\text {scalar }}=-\frac{1}{2} \partial_{\mu} \phi \partial_{\nu} \phi g^{\mu \nu} \sqrt{-g}-\frac{1}{12}(1+\Delta \xi) \phi^{2} R \sqrt{-g}-\frac{1}{4} h^{2} \phi^{2} \varphi^{2} \sqrt{-g} .
$$

At one loop order this coupling induces the following correction to the derivative of the inflaton potential (assuming, as always, that $\varphi$ is constant),

$$
\Delta V_{\text {scalar }}^{\prime}(\varphi)=\delta \xi R \varphi+\frac{1}{6} \delta \lambda \varphi^{3}+\frac{1}{2} h^{2} \varphi i \Delta\left[\xi, \frac{1}{2} h^{2} \varphi^{2}\right](x ; x) .
$$

The coincidence limit of the propagator is the primitive contribution, while $\delta \xi R \varphi$ represents a renormalization of the inflaton's (classically zero) conformal coupling and $\frac{1}{6} \delta \lambda \varphi^{3}$ renormalizes the inflaton's quartic self-coupling.

We specialize to de Sitter and choose the finite parts of the two counterterms to cancel the $\varphi^{2}$ and $\varphi^{4}$ terms in the small field expansion [29, 30],

$$
\begin{aligned}
& \delta \xi=-\frac{\Delta \xi h^{2} H^{D-4} \Gamma\left(1-\frac{D}{2}\right)}{12(4 \pi)^{\frac{D}{2}}}-\frac{\Delta \xi h^{2}}{192 \pi^{2}}\left[\psi\left(\nu_{+}\right)+\psi\left(\nu_{-}\right)\right] \\
& \delta \lambda=-\frac{3 h^{4} H^{D-4} \Gamma\left(1-\frac{D}{2}\right)}{2(4 \pi)^{\frac{D}{2}}}-\frac{3 \Delta \xi h^{4}}{16 \pi^{2}}\left[\frac{\psi\left(\nu_{+}\right)+\psi\left(\nu_{-}\right)}{2 \Delta \xi}-\frac{\left[\psi^{\prime}\left(\nu_{+}\right)-\psi^{\prime}\left(\nu_{-}\right)\right]}{\sqrt{1-8 \Delta \xi}}\right] .
\end{aligned}
$$

Here and henceforth $\psi(x) \equiv \frac{d}{d x} \ln [\Gamma(x)]$ is the digamma function, and we define $\nu_{ \pm} \equiv \frac{1}{2} \pm \frac{1}{2} \sqrt{1-8 \Delta \xi}$. The renormalized result can be expressed in terms of the dimensionless quantity $z \equiv h \varphi / H$ [29, 30],

$$
\begin{gathered}
\Delta V_{\text {scalar }}=\frac{H^{4}}{64 \pi^{2}}\left\{-\left[\psi\left(\nu_{+}\right)+\psi\left(\nu_{-}\right)\right]\left[2 \Delta \xi z^{2}+\frac{z^{4}}{4}\right]+\left[\psi^{\prime}\left(\nu_{+}\right)-\psi^{\prime}\left(\nu_{-}\right)\right] \frac{\frac{1}{2} \Delta \xi z^{4}}{\sqrt{1-8 \Delta \xi}}\right. \\
\left.+\int_{0}^{z^{2}} d x\left(2 \Delta \xi+\frac{x}{2}\right)\left[\psi\left(\frac{1}{2}+\sqrt{\frac{1}{4}-2 \Delta \xi-\frac{x}{2}}\right)+\psi\left(\frac{1}{2}-\sqrt{\frac{1}{4}-2 \Delta \xi-\frac{x}{2}}\right)\right]\right\} .
\end{gathered}
$$

The large field expansion comes from substituting in (86) the large argument expansion for the digamma function,

$$
|z| \gg 1 \Longrightarrow \psi(z)=\ln (z)-\frac{1}{2 z}-\frac{1}{12 z^{2}}+\frac{1}{120 z^{4}}-\frac{1}{256 z^{6}}+O\left(\frac{1}{z^{8}}\right) .
$$


The resulting expansion is,

$$
\begin{aligned}
& \Delta V_{\text {scalar }}=\frac{H^{4}}{64 \pi^{2}}\left\{\frac{1}{4} z^{4} \ln \left(\frac{1}{2} z^{2}+2 \Delta \xi\right)-\left[\frac{1}{8}+\frac{\left[\psi\left(\nu_{+}\right)+\psi\left(\nu_{-}\right)\right]}{4}\right.\right. \\
& \left.-\frac{\Delta \xi\left[\psi^{\prime}\left(\nu_{+}\right)-\psi^{\prime}\left(\nu_{-}\right)\right]}{2 \sqrt{1-8 \Delta \xi}}\right] z^{4}+2 \Delta \xi z^{2} \ln \left(\frac{1}{2} z^{2}+2 \Delta \xi\right)-\left[\frac{1}{3}+\Delta \xi\right. \\
& \left.\left.+2 \Delta \xi\left[\psi\left(\nu_{+}\right)+\psi\left(\nu_{-}\right)\right]\right] z^{2}+\left[4 \Delta \xi^{2}-\frac{2}{15}\right] \ln \left(\frac{1}{2} z^{2}+2 \Delta \xi\right)+O\left(z^{0}\right)\right\} .
\end{aligned}
$$

Because taking $H$ to zero makes $z=h \varphi / H$ large, it is this form (10) which makes contact with the famous Coleman-Weinberg potential of flat space [31]. To get the small field expansion we Taylor expand the digamma functions in expression (8) and integrate,

$$
\begin{gathered}
\Delta V_{\text {scalar }}=\frac{H^{4}}{64 \pi^{2}}\left\{\left[\frac{(1-6 \Delta \xi)\left[-\psi^{\prime}\left(\nu_{+}\right)+\psi^{\prime}\left(\nu_{-}\right)\right]}{(1-8 \Delta \xi)^{\frac{3}{2}}}+\frac{\Delta \xi\left[\psi^{\prime \prime}\left(\nu_{+}\right)+\psi^{\prime \prime}\left(\nu_{-}\right)\right]}{1-8 \Delta \xi}\right] \frac{z^{6}}{12}\right. \\
+\left[\frac{3(1-4 \Delta \xi)\left[-\psi^{\prime}\left(\nu_{+}\right)+\psi^{\prime}\left(\nu_{-}\right)\right]}{(1-8 \Delta \xi)^{\frac{5}{2}}}+\frac{3(1-4 \Delta \xi)\left[\psi^{\prime \prime}\left(\nu_{+}\right)+\psi^{\prime \prime}\left(\nu_{-}\right)\right]}{2(1-8 \Delta \xi)^{2}}\right. \\
\left.\left.+\frac{\Delta \xi\left[-\psi^{\prime \prime \prime}\left(\nu_{+}\right)+\psi^{\prime \prime \prime}\left(\nu_{-}\right)\right]}{(1-8 \Delta \xi)^{\frac{3}{2}}}\right] \frac{z^{8}}{96}+O\left(z^{10}\right)\right\} .(11)
\end{gathered}
$$

\subsection{Coupling to a massless fermion}

The inflaton might be Yukawa-coupled to a massless Dirac fermion $\psi_{i}(x)$

$$
\mathcal{L}_{\text {fermion }}=\bar{\psi} \gamma^{b} e^{\mu}{ }_{b}\left(\partial_{\mu}+\frac{i}{2} A_{\mu c d} J^{c d}\right) \psi \sqrt{-g}-f \varphi \bar{\psi} \psi \sqrt{-g} .
$$

Here $e^{\mu}{ }_{b}(x)$ is the vierbein field with $g^{\mu \nu}(x)=e^{\mu}{ }_{b}(x) e^{\nu}{ }_{c}(x) \eta^{b c}$ and $A_{\mu c d}(x)=$ $e^{\nu}{ }_{c}\left[e_{\nu d, \mu}-\Gamma_{\mu \nu}^{\rho} e_{\rho d}\right]$ is the spin connection. The symbol $\gamma_{i j}^{b}$ represents the $4 \times 4$ gamma matrices which obey $\left\{\gamma^{b}, \gamma^{c}\right\}=-2 \eta^{b c} I$, and $J^{c d} \equiv \frac{i}{4}\left[\gamma^{c}, \gamma^{d}\right]$ are the Lorentz representation matrices for Dirac fermions.

For cosmology we only require the fermion propagator for a general homogeneous, isotropic and spatially flat geometry in conformal coordinates,

$$
d s^{2}=a^{2}(\eta)\left[-d \eta^{2}+d \vec{x} \cdot d \vec{x}\right] \quad e_{\mu b}(x)=a(\eta) \eta_{\mu b} \quad H(\eta) \equiv \frac{a^{\prime}}{a^{2}} \quad \epsilon(\eta) \equiv-\frac{H^{\prime}}{a H^{2}},
$$


where $H(\eta)$ is the Hubble parameter and $\epsilon(\eta)$ is the first slow roll parameter, which is assumed to lie in the range $0 \leq \epsilon<1$. In the cosmological geometry (13) the appropriate fermion propagator is [32, 33],

$$
\begin{aligned}
i\left[{ }_{i} S_{j}\right]\left(x ; x^{\prime}\right) & =\frac{1}{a^{\frac{D+1}{2}}(\eta)}\left[i \gamma^{\mu} \partial_{\mu}+a(\eta) m I\right] \frac{a^{\frac{D-1}{2}}(\eta)}{\sqrt{a(\eta) a\left(\eta^{\prime}\right)}} \\
& \times\left\{i \Delta\left[\xi_{c}, M_{+}^{2}\right]\left(x ; x^{\prime}\right)\left(\frac{I+\gamma^{0}}{2}\right)+i \Delta\left[\xi_{c}, M_{-}^{2}\right]\left(x ; x^{\prime}\right)\left(\frac{I-\gamma^{0}}{2}\right)\right\},
\end{aligned}
$$

where $\xi_{c} \equiv \frac{1}{2(D-1)}$ and $M_{ \pm}^{2} \equiv f \varphi(f \varphi \mp i H)$.

At one loop order the coupling (12) induces the following correction to the $\varphi$ derivative of the inflaton potential,

$$
\Delta V_{\text {fermion }}^{\prime}(\varphi)=\delta \xi \varphi R+\frac{1}{6} \delta \lambda \varphi^{3}-f i\left[{ }_{i} S_{i}\right](x ; x) .
$$

Of course the trace of the coincident fermion propagator is the primitive contribution, while the terms proportional to $\delta \xi$ and $\delta \lambda$ are counterterms. Specializing to de Sitter (hence $H(\eta)$ constant) and renormalizing so as to null the quadratic and quartic terms in the small field expansion [33],

$$
\begin{aligned}
\delta \xi & =\frac{4 f^{2} H^{D-4}}{(4 \pi)^{\frac{D}{2}}} \frac{\Gamma\left(1-\frac{D}{2}\right)}{D(D-1)}+\frac{(1-\gamma) f^{2}}{24 \pi^{2}}, \\
\delta \lambda & =\frac{24 f^{4} H^{D-4}}{(4 \pi)^{\frac{D}{2}}} \Gamma\left(1-\frac{D}{2}\right)+\frac{3[\zeta(3)-\gamma] f^{4}}{\pi^{2}},
\end{aligned}
$$

gives rise to the following renormalized result [32, 33],

$$
\begin{aligned}
& \Delta V_{\text {fermion }}(\varphi)=-\frac{H^{4}}{8 \pi^{2}}\left\{2 \gamma\left(\frac{f \varphi}{H}\right)^{2}-[\zeta(3)-\gamma]\left(\frac{f \varphi}{H}\right)^{4}\right. \\
&\left.+2 \int_{0}^{\frac{f \varphi}{H}} d x\left(x+x^{3}\right)[\psi(1+i x)+\psi(1-i x)]\right\} .
\end{aligned}
$$

Substituting (91) in (18) gives the large field expansion with $z \equiv f \varphi / H$,

$$
\begin{aligned}
\Delta V_{\text {fermion }}=-\frac{H^{4}}{8 \pi^{2}}\left\{\frac{1}{2} z^{4} \ln \left(z^{2}+1\right)\right. & -\left(\zeta(3)+\frac{1}{4}-\gamma\right) z^{4}+z^{2} \ln \left(z^{2}+1\right) \\
& \left.-\left(\frac{4}{3}-2 \gamma\right) z^{2}+\frac{11}{60} \ln \left(z^{2}+1\right)+O\left(z^{0}\right)\right\} .
\end{aligned}
$$


As before, the Coleman-Weinberg form [31] is apparent in the leading large $z$ behavior. To get the small field expansion we substitute,

$$
|z| \ll 1 \Longrightarrow \psi(1+z)=-\gamma-\sum_{k=1}^{\infty} \zeta(k+1)(-z)^{k} .
$$

The resulting expansion is,

$$
\begin{aligned}
\Delta V_{\text {fermion }} & =-\frac{H^{4}}{4 \pi^{2}} \sum_{n=2}^{\infty} \frac{(-1)^{n}}{n+1}[\zeta(2 n-1)-\zeta(2 n+1)] z^{2 n+2} \\
& =-\frac{H^{4}}{8 \pi^{2}}\left\{\frac{2}{3}[\zeta(3)-\zeta(5)] z^{6}-\frac{1}{2}[\zeta(5)-\zeta(7)] z^{8}+O\left(z^{10}\right)\right\} .
\end{aligned}
$$

\subsection{Coupling to a vector gauge boson}

A complex inflaton might couple to electromagnetism,

$$
\mathcal{L}_{\text {vector }}=-\frac{1}{4} F_{\rho \sigma} F_{\mu \nu} g^{\rho \mu} g^{\sigma \nu} \sqrt{-g}-\left(\partial_{\mu}-i e A_{\mu}\right) \varphi\left(\partial_{\nu}+i e A_{\nu}\right) \varphi^{*} g^{\mu \nu} \sqrt{-g} .
$$

Here $A_{\mu}(x)$ is the vector potential and $F_{\mu \nu} \equiv \partial_{\mu} A_{\nu}-\partial_{\nu} A_{\mu}$ is the field strength tensor. At our one loop level the result for a non-Abelian charged scalar would be proportional to the electromagnetic result derived from (23).

Coleman and Weinberg noted that Lorentz gauge $\left(\partial_{\mu}\left[\sqrt{-g} g^{\mu \nu} A_{\nu}\right]=0\right)$ makes the coupling $i e A_{\mu}\left[\varphi_{, \nu}^{*} \varphi-\varphi^{*} \varphi_{, \nu}\right] g^{\mu \nu} \sqrt{-g}$ drop from the one loop effective potential [31]. Hence we employ the transverse vector propagator [34,

$$
\left.\left[\square^{\mu \nu}-R^{\mu \nu}-M_{V}^{2} g^{\mu \nu}\right] i{ }_{\nu} \Delta_{\rho}\right]\left(x ; x^{\prime}\right)=\frac{i \delta_{\rho}^{\mu} \delta^{D}\left(x-x^{\prime}\right)}{\sqrt{-g}}+g^{\mu \nu} \partial_{\nu} \partial_{\rho}^{\prime} i \Delta[0,0]\left(x ; x^{\prime}\right)
$$

where $\square^{\mu \nu}$ is the covariant vector d'Alembertian and $M_{V}^{2}=2 e^{2} \varphi^{*} \varphi$. For de Sitter (and we conjecture generally) it is best to express this propagator as 2nd order transverse projectors on $x^{\mu}$ and $x^{\prime \mu}$, contracted into an invariant 
bi-vector multiplied by a scalar structure function [35],

$$
\begin{aligned}
\left.i{ }_{\mu} \Delta_{\rho}\right]\left(x ; x^{\prime}\right) & =\mathbf{P}_{\mu}^{\nu}(x) \times \mathbf{P}_{\rho}^{\sigma}\left(x^{\prime}\right) \times\left[\frac{\partial^{2} \ell^{2}\left(x ; x^{\prime}\right)}{\partial x^{\nu} \partial x^{\prime}} \times \mathcal{S}\left(x ; x^{\prime}\right)\right] \\
\mathbf{P}_{\mu}^{\nu}(x) & \equiv \mathbf{\square}_{\mu}^{\nu}-D^{\nu} D_{\mu} \\
\mathcal{S}\left(x ; x^{\prime}\right) & \equiv \frac{i \Delta\left[\xi_{v}, M_{V}^{2}\right]-i \Delta\left[\xi_{v}, 0\right]}{M_{V}^{4}}-\left.\frac{1}{M_{V}^{2}} \frac{\partial i \Delta\left[\xi_{v}, N^{2}\right]}{\partial N^{2}}\right|_{N^{2}=0} .
\end{aligned}
$$

Here $D_{\mu}$ is the covariant derivative operator, $\xi_{v} \equiv \frac{(D-2)}{D(D-1)}$ and $\ell^{2}\left(x ; x^{\prime}\right)$ is some function of the invariant length from $x^{\mu}$ to $x^{\prime \mu}$.

The photon loop contribution to the inflaton potential takes the form,

$$
\Delta V_{\text {vector }}^{\prime}\left(\varphi^{*} \varphi\right)=\delta \xi R+\frac{1}{2} \delta \lambda \varphi^{*} \varphi+e^{2} g^{\mu \nu} i\left[{ }_{\mu} \Delta_{\nu}\right](x ; x) .
$$

As before, we choose the conformal and quartic renormalizations to cancel the $\varphi^{*} \varphi$ and $\left(\varphi^{*} \varphi\right)^{2}$ terms in the small field expansion of $V_{\text {vector }}\left(\varphi^{*} \varphi\right)$ [36],

$$
\begin{aligned}
\delta \xi & =\frac{e^{2} H^{D-4}}{(4 \pi)^{\frac{D}{2}}}\left\{\frac{1}{4-D}+\frac{\gamma}{2}+O(D-4)\right\} \\
\delta \lambda & =\frac{D(D-1) e^{4} H^{D-4}}{(4 \pi)^{\frac{D}{2}}}\left\{\frac{2}{4-D}+\gamma-\frac{3}{2}+O(D-4)\right\} .
\end{aligned}
$$

The final renormalized result is [36, 37],

$$
\begin{aligned}
\Delta V_{\text {vector }}= & \frac{3 H^{4}}{8 \pi^{2}}\left\{(-1+2 \gamma) z^{2}+\left(-\frac{3}{2}+\gamma\right) z^{4}\right. \\
& \left.+\int_{0}^{z^{2}} d x(1+x)\left[\psi\left(\frac{3}{2}+\frac{1}{2} \sqrt{1-8 x}\right)+\psi\left(\frac{3}{2}-\frac{1}{2} \sqrt{1-8 x}\right)\right]\right\},
\end{aligned}
$$

where we define $z^{2} \equiv \frac{e^{2} \varphi^{*} \varphi}{H^{2}}$.

The large field expansion derives from substituting (9) in (31),

$$
\begin{array}{r}
\Delta V_{\text {vector }}=\frac{3 H^{4}}{8 \pi^{2}}\left\{\frac{1}{2} z^{4} \ln \left(z^{2}+1\right)+\left[-\frac{7}{4}+\frac{1}{2} \ln (2)+\gamma\right] z^{4}+z^{2} \ln \left(z^{2}+1\right)\right. \\
\left.+\left[-\frac{13}{6}+\ln (2)+2 \gamma\right] z^{2}+\frac{19}{60} \ln \left(z^{2}+1\right)+O\left(z^{0}\right)\right\} .
\end{array}
$$


As before, the leading large $z$ form agrees with the flat space result derived by Coleman and Weinberg [31. Substituting (20) in (31) and performing the integral gives the small field expansion,

$$
\begin{aligned}
& \Delta V_{\text {vector }}=\frac{3 H^{4}}{8 \pi^{2}}\left\{\frac{1}{2} \ln (1-\Delta z)+\frac{1}{2} \Delta z+\frac{1}{4} \Delta z^{2}+\frac{7}{12} \Delta z^{3}-\frac{3}{8} \Delta z^{4}\right. \\
& \left.\quad+\sum_{m=1}^{\infty} \zeta(2 m+1)\left[-\frac{\Delta z^{2 m+1}}{2 m+1}+\frac{\frac{3}{2} \Delta z^{2 m+2}}{2 m+2}+\frac{\frac{3}{2} \Delta z^{2 m+3}}{2 m+3}-\frac{\Delta z^{2 m+4}}{2 m+4}\right]\right\},
\end{aligned}
$$

where we define $\Delta z$ as,

$$
\Delta z \equiv \frac{1}{2}-\frac{1}{2} \sqrt{1-8 z^{2}}=2 z^{2}+4 z^{4}+16 z^{6}+80 z^{8}+O\left(z^{10}\right) .
$$

Substituting (34) into (33) gives,

$$
\Delta V_{\text {vector }}=\frac{3 H^{4}}{8 \pi^{2}}\left\{\left(\frac{10}{3}-\frac{8}{3} \zeta(3)\right) z^{6}+(12-10 \zeta(3)) z^{8}+O\left(z^{10}\right)\right\}
$$

\section{What Is $H$ Generally?}

In section 2 we saw that various matter couplings to the inflaton induce corrections to the inflaton potential which on de Sitter take the general form,

$$
\left.\Delta V\right|_{\text {de Sitter }}=H^{4} f\left(z^{2}\right) \quad, \quad z^{2} \propto \frac{1}{H^{2}}
$$

where $z^{2}$ is the norm-squared of the coupling constant times the inflaton divided by $H$. If inflation were exactly de Sitter then $H(\eta)$ would be a constant and we could eliminate all or part of $\Delta V$ by fine tuning the classical potential. However, $H(\eta)$ must change in any realistic model of inflation, and this calls into question what functional of the metric those factors of " $H$ " really are, at least for a general inflationary background of the form (13). We cannot answer this question by direct computation because the scalar propagator $i \Delta\left[\xi, M^{2}\right]\left(x ; x^{\prime}\right)$ is not known for geometries other than de Sitter (and flat space) when $M^{2} \neq 0$. We shall instead use indirect arguments to

conclude first, that most of the factors of $H$ are dynamical functionals of the metric and second, that these functionals cannot even be local. 
First, consider the contribution to the stress tensor from whatever coupling (scalar, fermion or vector) we choose,

$$
\Delta T_{\mu \nu}(x) \equiv-\frac{2}{\sqrt{-g(x)}} \frac{\delta S_{\text {coupling }}}{\delta g^{\mu \nu}(x)} .
$$

We can evaluate the expectation value of $\Delta T_{\mu \nu}$ for the de Sitter geometry, in the appropriate matter vacuum, and use this to probe how the factors of $H$ depend upon the metric. For example, if all the factors of $H$ are constant then we would find,

$$
H^{2}=\frac{1}{3} \Lambda \quad \Longrightarrow \quad\left\langle\Delta T_{\mu \nu}\right\rangle_{\text {de Sitter }}=-g_{\mu \nu} H^{4} \times \frac{1}{2} f\left(z^{2}\right) .
$$

If the factors of $H$ derive from the Ricci scalar, we would find,

$$
H^{2}=\frac{1}{12} R \quad \Longrightarrow \quad\left\langle\Delta T_{\mu \nu}\right\rangle_{\text {de Sitter }}=-g_{\mu \nu} H^{4} \times \frac{1}{2} z^{2} f^{\prime}\left(z^{2}\right) .
$$

The presence in this expression of $f^{\prime}\left(z^{2}\right)$, rather than $f\left(z^{2}\right)$, originates from the metric variation of $R$.

The actual result is a combination of both possibilities (38) and (39) [30, 33, 36], although the predominant behavior is (39). Only the factors of $H^{D-4}$ which were introduced in the renormalization counterterms (6-77), (1617) and (29) 30) are consistent with being constants. Changing them from $H$ to an arbitrary renormalization scale $\mu$ effects the following changes in the de Sitter effective potentials,

$$
\begin{aligned}
\Delta V_{\text {scalar }} & \longrightarrow \Delta V_{\text {scalar }}+\frac{H^{4}}{64 \pi^{2}}\left[\frac{1}{4} z^{4}+2 \Delta \xi z^{2}\right] \ln \left(\frac{H^{2}}{\mu^{2}}\right), \\
\Delta V_{\text {fermion }} & \longrightarrow \Delta V_{\text {fermion }}-\frac{H^{4}}{8 \pi^{2}}\left[\frac{1}{2} z^{4}+z^{2}\right] \ln \left(\frac{H^{2}}{\mu^{2}}\right), \\
\Delta V_{\text {vector }} & \longrightarrow \Delta V_{\text {vector }}+\frac{3 H^{4}}{8 \pi^{2}}\left[\frac{1}{2} z^{4}+z^{2}\right] \ln \left(\frac{H^{2}}{\mu^{2}}\right) .
\end{aligned}
$$

From expressions (10), (19) and (32) we see that these replacements remove the logarithmic dependence upon $H^{2}$ from the $z^{4} \ln \left(z^{2}\right)$ and $z^{2} \ln \left(z^{2}\right)$ terms of the large field expansions, leaving only the $\ln \left(z^{2}\right)$ term 1

\footnotetext{
${ }^{1}$ Recall that $z^{2}$ contains the inverse of $H^{2}$, so the factors of $\ln \left(z^{2}\right)$ in expressions (10), (19) and (32) go like $-\ln \left(H^{2}\right)=-\ln \left(\mu^{2}\right)-\ln \left(H^{2} / \mu^{2}\right)$.
} 
The remaining factors of $H^{2}$ are unknown functionals of the metric which have the property that their first variations are indistinguishable from that of $\frac{1}{12} R$ for de Sitter. This leaves a vast number of possibilities. One can think of doing a sort of functional Taylor expansion about de Sitter, and of course fixing the 0th and 1st order terms in no way constrains the remaining ones.

It is easy to see that the remaining factors of $H^{2}$ are not local functionals of the metric. Note first that it is the small $\varphi$ expansion which matters for inflation. 2 Hence we can expand the propagator in powers of $\varphi$, which is also the mass of our general scalar propagator (2),

$$
\begin{gathered}
i \Delta\left[\xi, M^{2}\right](x ; x)=i \Delta[\xi, 0](x ; x)-i M^{2} \int d^{D} w \sqrt{-g(w)}(i \Delta[\xi, 0](x ; w))^{2} \\
+\sum_{n=2}^{\infty}\left(-i M^{2}\right)^{n} \int d^{D} w_{1} \sqrt{-g\left(w_{1}\right)} i \Delta[\xi, 0]\left(x ; w_{1}\right) \int d^{D} w_{2} \sqrt{-g\left(w_{2}\right)} \\
\quad \times i \Delta[\xi, 0]\left(w_{1} ; w_{2}\right) \ldots \int d^{D} w_{n} i \Delta[\xi, 0]\left(w_{n-1} ; w_{n}\right) i \Delta[\xi, 0]\left(w_{n} ; x\right) .
\end{gathered}
$$

Although we cannot solve for $i \Delta[\xi, 0]\left(x ; x^{\prime}\right)$ for a general cosmological geometry (13), it is known for the infinite sub-class of geometries with constant $\epsilon(\eta)$ [38]. Except for $\epsilon(\eta)=0$ (de Sitter), these geometries interpolate between different values of $H(\eta)$. With expression (43) this is already enough to see that the factors of " $H^{2}$ " are not even local.

\section{Conclusions}

The increasingly tight upper bounds on the tensor-to-scalar ratio have heightened the unease that many feel over the degree of fine tuning which is required to make scalar potential models (11) conform to the data [25, 26, 27, 28]. But it has hitherto been believed that fine tuning would at least suffice, however distasteful it might be. We have argued here that there may be a fundamental obstacle associated with quantum corrections to the inflaton potential from the matter couplings which are needed for reheating. Contrary to the simple $\varphi^{4} \ln \left(\varphi^{2}\right)$ effective potentials which are induced in flat space [31], the de Sitter space results for loops of other scalars (8), massless fermions (18) and vectors (31) all exhibit a complicated dependence on the dimensionless

\footnotetext{
${ }^{2}$ If $\varphi$ had a large field minimum we would simply have subtracted this off in the couplings (4), (12) and (23).
} 
ratio of the inflaton to the inflationary Hubble parameter. By considering the expectation value of the stress tensor specialized to de Sitter we showed that most of these factors cannot be actual constants but must rather be dynamical functionals of the metric which change with time as any realistic model of inflation evolves. It is only the leading term of the large field expansions (10), (19) and (32), which recovers the $H$-independent Coleman-Weinberg form. If the factors of $H^{2}$ which appear in the rest of the effective potential were local - for example, $H^{2} \rightarrow \frac{1}{12} R$, which is consistent with the de Sitter stress tensor results [30, 33, 36] - then any undesirable effects could still be fine tuned away. However, we argued in section 3 that the dependence on the metric cannot be local, even when specialized to a cosmological background (13). This means that no local counterterm can fully eliminate quantum corrections to the inflaton potential during actual inflation.

More study is needed to quantify how bad the problem actually is. For example, if undesirable terms in $\Delta V$ are eliminated at one instant in time using a local counterterm - either with the replacement $H^{2} \rightarrow \frac{1}{3} \Lambda$ or $H^{2} \rightarrow$ $\frac{1}{12} R$ - how large does the residual effect at other times become during the $\sim 50$ e-foldings of primordial inflation which are required to solve the horizon problem? Although the problem cannot be solved exactly because we only know the key propagator $i \Delta\left[\xi, M^{2}\right]\left(x ; x^{\prime}\right)$ for de Sitter, it might be reduced to a series of conformal time integrations using the expansion (43) for the class of constant $\epsilon(\eta)$ geometries $3^{3}$ Because the matter theories (44), (12) and (23) are all renormalizable, the terms at order $M^{6}$ and higher must be ultraviolet finite, so they can be evaluated with $D=4$, and the resulting temporal integrations could at least be performed numerically for specific values of $\epsilon$.

We expect that the constant $\epsilon$ study would reveal deficiencies with the replacement $H^{2} \rightarrow \frac{1}{12} R$ which could be repaired with a better local replacement of the form $H^{2} \rightarrow \frac{1}{12} f(\epsilon) R$, where " $\epsilon$ " can be reconstructed from ratios of curvatures using the constant $\epsilon$ relations,

$$
\begin{aligned}
R \longrightarrow 6(2-\epsilon) H^{2} & , \quad R^{\mu \nu} R_{\mu \nu} \longrightarrow 12\left(3-3 \epsilon+\epsilon^{2}\right) H^{4}, \\
\square R \longrightarrow 36 \epsilon(1-\epsilon)(2-\epsilon) H^{4} & , \quad R^{\mu \nu \rho \sigma} R_{\mu \nu \rho \sigma} \longrightarrow 12\left(2-2 \epsilon+\epsilon^{2}\right) H^{4} .
\end{aligned}
$$

Had we not been assuming $\dot{\epsilon}=0$ the expression for $\square R$ would have involved the second slow roll parameter $\eta=\epsilon-\dot{\epsilon} / 2 H \epsilon$, which can be much larger than $\epsilon$. It is therefore safer to infer " $\epsilon$ " from the other curvature scalars, for

\footnotetext{
${ }^{3}$ The Schwinger-Keldysh formalism 39, 40, 41, 42, 43, 44, 45, 46, 47, 48, 49, would be necessary to provide a real and causal form for (43).
} 
example,

$$
\frac{2}{2-\epsilon} \longrightarrow 1+\sqrt{1-\frac{6 \mathcal{G}}{R^{2}}},
$$

where $\mathcal{G} \equiv R^{\mu \nu \rho \sigma} R_{\mu \nu \rho \sigma}-4 R^{\mu \nu} R_{\mu \nu}+R^{2}$ is the Gauss-Bonnet scalar. Of course higher curvatures beyond $F(R)$ models would suffer the Ostrogradskian instability and are not permitted in fundamental theory [14].

The data on $n_{s}$ and $r$ are now inconsistent with any constant $\epsilon$ model so even the unacceptable higher curvature terms will not suffice. However, we should quantify the deviations between the best local constant $\epsilon$ subtraction and the actual result of $i \Delta\left[\xi, M^{2}\right](x ; x)$ for nonconstant $\epsilon$. The tree order power spectrum contains a truly nonlocal "memory effect" which is not recovered by the constant $\epsilon$ result [50], and the same must be true for $i \Delta\left[\xi, M^{2}\right](x ; x)$ as well.

Recent progress on expressing the mode functions for general $\epsilon(t)$ holds out the prospect of being able to develop a comparably effective approximation of the relevant propagators [51]. It is also worthwhile examining the possibility of synergies between the infrared results we have for de Sitter and the local expansions which have recently been applied to Higgs inflation [52, 53, 54. It may be that combining the two methods will reveal more about what " $H^{2}$ " is generally than either does by itself.

A final observation is that these matter loop corrections to the inflaton effective potential might also modify gravity at late times because the metric dependence in " $H^{2}$ " is not Planck-suppressed. We need to quantify how large the residual effect can be after the best allowable counterterm has been removed. In particular, one needs to understand whether or not the nonlocality of the primitive contributions can result in some very high scale from early times surviving to affect late time physics.

\section{Acknowledgements}

We have benefited from conversations with R. H. Brandenberger, T. Prokopec and N. C. Tsamis. This work was partially supported by Taiwan MOST grant 103-2112-M-006-001-MY3, by NSF grants PHY-1205591 and PHY-1506513, and by the UF's Institute for Fundamental Theory.

\section{References}

[1] R. Brout, F. Englert and E. Gunzig, Annals Phys. 115, 78 (1978). 
[2] A. A. Starobinsky, Phys. Lett. B 91, 99 (1980).

[3] D. Kazanas, Astrophys. J. 241, L59 (1980).

[4] K. Sato, Mon. Not. Roy. Astron. Soc. 195, 467 (1981).

[5] A. H. Guth, Phys. Rev. D 23, 347 (1981).

[6] W. H. Kinney, G. Geshnizjani and A. M. Dizgah, in Proceedings the XLVIIth Recontres de Moriond (ARISF, Paris, 2012), ed. E. Augè, J. Dumarchez and J. T. T. Vân, p. 179.

[7] R. P. Woodard, Rept. Prog. Phys. 72, 126002 (2009) arXiv:0907.4238 [gr-qc]].

[8] A. D. Linde, Phys. Lett. B 108, 389 (1982).

[9] A. Albrecht and P. J. Steinhardt, Phys. Rev. Lett. 48, 1220 (1982).

[10] A. D. Linde, Phys. Lett. B 129, 177 (1983).

[11] N. C. Tsamis and R. P. Woodard, Annals Phys. 267, 145 (1998) hep-ph/9712331.

[12] T. D. Saini, S. Raychaudhury, V. Sahni and A. A. Starobinsky, Phys. Rev. Lett. 85, 1162 (2000) astro-ph/9910231.

[13] S. Capozziello, S. Nojiri and S. D. Odintsov, Phys. Lett. B 634, 93 (2006) hep-th/0512118.

[14] R. P. Woodard, Lect. Notes Phys. 720, 403 (2007) astro-ph/0601672.

[15] Z. K. Guo, N. Ohta and Y. Z. Zhang, Mod. Phys. Lett. A 22, 883 (2007) astro-ph/0603109.

[16] D. S. Goldwirth and T. Piran, Phys. Rev. Lett. 64, 2852 (1990).

[17] D. S. Goldwirth and T. Piran, Phys. Rept. 214, 223 (1992).

[18] T. Vachaspati and M. Trodden, Phys. Rev. D 61, 023502 (1999) gr-qc/9811037.

[19] V. F. Mukhanov, H. A. Feldman and R. H. Brandenberger, Phys. Rept. 215, 203 (1992). 
[20] R. Allahverdi, R. Brandenberger, F. Y. Cyr-Racine and A. Mazumdar, Ann. Rev. Nucl. Part. Sci. 60, 27 (2010) [arXiv:1001.2600 [hep-th]].

[21] A. G. Riess et al. [Supernova Search Team Collaboration], Astron. J. 116, 1009 (1998) [astro-ph/9805201].

[22] S. Perlmutter et al. [Supernova Cosmology Project Collaboration], Astrophys. J. 517, 565 (1999) astro-ph/9812133.

[23] Y. Wang and P. Mukherjee, Astrophys. J. 650, 1 (2006) astro-ph/0604051.

[24] U. Alam, V. Sahni and A. A. Starobinsky, JCAP 0702, 011 (2007) astro-ph/0612381.

[25] A. Ijjas, P. J. Steinhardt and A. Loeb, Phys. Lett. B 723, 261 (2013) arXiv:1304.2785 [astro-ph.CO]].

[26] A. H. Guth, D. I. Kaiser and Y. Nomura, Phys. Lett. B 733, 112 (2014) arXiv:1312.7619 [astro-ph.CO]].

[27] A. Linde, arXiv:1402.0526 [hep-th].

[28] A. Ijjas, P. J. Steinhardt and A. Loeb, Phys. Lett. B 736, 142 (2014) [arXiv:1402.6980 [astro-ph.CO]].

[29] A. Bilandzic and T. Prokopec, Phys. Rev. D 76, 103507 (2007) arXiv:0704.1905 [astro-ph]].

[30] T. M. Janssen, S. P. Miao, T. Prokopec and R. P. Woodard, JCAP 0905, 003 (2009) arXiv:0904.1151 [gr-qc]].

[31] S. R. Coleman and E. J. Weinberg, Phys. Rev. D 7, 1888 (1973).

[32] P. Candelas and D. J. Raine, Phys. Rev. D 12, 965 (1975).

[33] S. P. Miao and R. P. Woodard, Phys. Rev. D 74, 044019 (2006) gr-qc/0602110].

[34] N. C. Tsamis and R. P. Woodard, J. Math. Phys. 48, 052306 (2007) gr-qc/0608069. 
[35] S. P. Miao, N. C. Tsamis and R. P. Woodard, J. Math. Phys. 52, 122301 (2011) arXiv:1106.0925 [gr-qc]].

[36] T. Prokopec, N. C. Tsamis and R. P. Woodard, Annals Phys. 323, 1324 (2008) arXiv:0707.0847 [gr-qc]].

[37] B. Allen, Nucl. Phys. B 226, 228 (1983).

[38] T. M. Janssen, S. P. Miao, T. Prokopec and R. P. Woodard, Class. Quant. Grav. 25, 245013 (2008) [arXiv:0808.2449 [gr-qc]].

[39] J. S. Schwinger, J. Math. Phys. 2, 407 (1961).

[40] K. T. Mahanthappa, Phys. Rev. 126, 329 (1962).

[41] P. M. Bakshi and K. T. Mahanthappa, J. Math. Phys. 4, 1 (1963).

[42] P. M. Bakshi and K. T. Mahanthappa, J. Math. Phys. 4, 12 (1963).

[43] L. V. Keldysh, Zh. Eksp. Teor. Fiz. 47, 1515 (1964) [Sov. Phys. JETP 20, 1018 (1965)].

[44] K. c. Chou, Z. b. Su, B. l. Hao and L. Yu, Phys. Rept. 118, 1 (1985).

[45] R. D. Jordan, Phys. Rev. D 33, 444 (1986).

[46] E. Calzetta and B. L. Hu, Phys. Rev. D 35, 495 (1987).

[47] E. Calzetta and B. L. Hu, Phys. Rev. D 37, 2878 (1988).

[48] S. Weinberg, Phys. Rev. D 72, 043514 (2005) hep-th/0506236].

[49] S. Weinberg, Phys. Rev. D 74, 023508 (2006) hep-th/0605244.

[50] L. M. Wang, V. F. Mukhanov and P. J. Steinhardt, Phys. Lett. B 414, 18 (1997) astro-ph/9709032.

[51] D. J. Brooker, N. C. Tsamis and R. P. Woodard, arXiv:1507.07452 [astro-ph.CO].

[52] A. O. Barvinsky, A. Y. Kamenshchik and A. A. Starobinsky, JCAP 0811, 021 (2008) [arXiv:0809.2104 [hep-ph]]. 
[53] A. O. Barvinsky, A. Y. Kamenshchik, C. Kiefer, A. A. Starobinsky and C. F. Steinwachs, Eur. Phys. J. C 72, 2219 (2012) arXiv:0910.1041 [hep-ph]].

[54] F. Bezrukov, A. Magnin, M. Shaposhnikov and S. Sibiryakov, JHEP 1101, 016 (2011) [arXiv:1008.5157 [hep-ph]]. 